\title{
Dioses indígenas vinculados a núcleos de población en la Hispania romana
}

\author{
Juan Carlos Olivares *
}

\begin{abstract}
RESUMEN
Una de las principales características de las ofrendas votivas a "Bandua" en Hispania es que se alude al dios con apelativos que le vinculan a núcleos de población, preferentemente «uici», "pagi" o "castella", mientras que los sobrenombres referentes a municipios

o capitales de "ciuitates" suelen llevarlos los Genios. En las provincias galas, los epítetos alusivos a comunidades locales acompañan, sobre todas las demás divinidades, a

Marte. "Bandua" fue, por tanto, un dios de carácter guerrero, protector y defensor de las comunidades indígenas que, después de la conquista romana, quedaría como divinidad tutelar del grupo social residente en el núcleo poblacional.
\end{abstract}

\section{ABSTRACT}

One of the main features of the votive offerings to "Bandua" in Hispania is that they allude to the god using certain epithets which link him to population centres, especially "uici", "pagi" or "castella". On the other hand, the appellations refering to towns or "ciuitates" capitals are usually born by the "Genii". In Galia, the epithets alluding to local communities refer to Mars, above all other gods. Thus, "Bandua" was a warrior-like god who acted as protector and defender of the indigenous cities and who, after the Roman conquest, would be the guardian deity for the social group living in the population centre.

* Universidad de Alicante. 
La relación que las comunidades de la Hispania indoeuropea tuvieron con determinadas divinidades puede ser analizada teniendo en cuenta datos de diversos tipos. A primera vista, el hallazgo de altares votivos dedicados a dioses indígenas en el interior de ciudades podría apuntar una especial vinculación de la divinidad con el conjunto de sus habitantes; sin embargo, la existencia en el interior de las mismas de unidades sociales más reducidas de carácter gentilicio nos impide, en buena medida, descubrir si la esfera funcional del dios en cuestión se relaciona con la comunidad ciudadana como tal, si afecta a un entorno más privado, familiar o clánico o si no se vincula a un grupo social concreto.

En algunos casos parece evidente la escasa utilidad de este dato, si no se considera junto a otras variables. A modo de ejemplo, en el enclave de Lucus Augusti conocemos varios teónimos masculinos indígenas como Laho, Reo, Verore o Regoni ${ }^{1}$. Por tanto, la constatación del culto a varias divinidades en el mismo enclave y, por otra parte, la alusión a ellas en ocasiones con sus categorías religiosas romanas nos impide identificar a una divinidad indígena que ejerciera su influencia concretamente sobre el conjunto social residente en el espacio urbano.

En otros enclaves donde la irrupción cultural de Roma no fue tan intensa, como los castros y, en general, en comunidades como uici, pagi o castella, podemos hallar nuevos datos, puesto que en estos enclaves se observa una yuxtaposición de elementos religiosos romanos e indígenas sin que, en muchos casos, se fusionen.

No siempre los investigadores relacionan los lugares donde se hallaron las inscripciones votivas con los núcleos habitados más o menos cercanos, pero tenemos alguna información al respecto. Lo primero que llama la atención es que son varios los posibles teónimos que aparecen en este tipo de contextos. Algunos ejemplos serían Caro, cerca de Castro de Roboreda (Santa Vaia do Río do Moinhos, Arcos de Valdevez, Vila Real) ${ }^{2}$; Reue, en Santa María de Castromao (Celanova, Orense) 3; el poco probable Saur, conocido por una inscripción en una pátera en la que se representaba una figura de Marte, junto al castro de Alvarelhos (Carriça,

\footnotetext{
1 Los teónimos serán citados en dativo, según aparecen en las inscripciones, exceptuando determinados teónimos muy extendidos que aparecen citados con ciertas variantes entre las distintas inscripciones. En estos casos, el teónimo lo mencionaremos en una sóla de sus formas como, por ejemplo, Bandua o Coso. En cuanto a Regoni, es posible que se trate de un epíteto cuyo teónimo se omitió en la inscripción.

2 VASCONCELOS, 1905, 336; ENCARNAÇÃO, 1975, 156-157.

3 FARINA, 1991, 57 ss.
} 
Santo Tirso, Porto) 4; Torolo, cerca de Castro Escuadro (Pías, Escuadro, Orense) ${ }^{5}$ o Coso, aunque la interpretación del texto es dudosa, a 500 metros del castro de Sanfins (Eiriz, Paços de Ferreira, Porto) ${ }^{6}$.

Sin duda el número de altares votivos hallados en el interior o las cercanías de estos núcleos urbanos se incrementará a medida que las excavaciones permitan una mayor contextualización de las piezas pero, según los datos de que disponemos en la actualidad, observamos que esta relación se repite con una cierta frecuencia en las dedicaciones a Bandua. En este sentido, podemos citar el ara de Castro do Mau Vizinho (Sul, S. Pedro do Sul, Viseu) dedicada a Bandua Oce...7, los tres altares dedicados a Bandua Roudaeco, hallados cerca del importante castro de Villavieja (Trujillo, Cáceres) ${ }^{8}$; el procedente de Eirás (San Amaro, Orense) cerca de los castros de Eirás y de "a Cibdá», muy cerca de San Ciprián de Las ${ }^{9}$ o los dos procedentes del castillo de Vila da Feira (Arlindo de Sousa, Aveiro) ${ }^{10}$.

Los datos de ese carácter no son muy numerosos y, por tanto, tampoco ofrecen conclusiones determinantes. Por otra parte, aunque se conociera una gran cantidad de inscripciones alusivas a un mismo dios en contextos urbanos, este hecho por sí mismo sería poco más que un indicio de difícil interpretación. Por tanto, hemos de calibrar otras variables que supongan un punto de partida más sólido para investigar la relación entre determinadas divinidades y los núcleos habitados indígenas.

\section{EPITETOS DE DIOSES VINCULABLES A NÚCLEOS DE POBLACIÓN EN EL TERRITORIO LUSITANO-GALAICO}

La epigrafía votiva del ámbito lusitano-galaico nos ofrece otros datos que son más importantes para los objetivos de nuestro estudio: se trata de los epítetos de divinidades indígenas que aluden, probablemente, a comunidades urbanas de distinto rango. Sobre ellos realizaremos un análisis más pormenorizado.

\footnotetext{
4 GaRcia, 1991, 527-528 n. ${ }^{\circ}$ 602. Alvarelhos sería un castro fuertemente romanizado (Alarcão, 1988,19 n. $\left.^{\circ} 348\right)$.

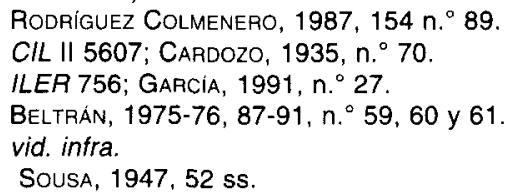


En primer lugar, hemos de tener en cuenta todos aquellos epítetos que contienen sufijos en -brigo, -breo o sus versiones en dativo pre-céltico que aludirían a topónimos en -briga, es decir, a enclaves urbanos predominantemente indígenas y fortificados ${ }^{11}$. Por otra parte, consideraremos aquellos sobrenombres que derivan de ciudades antiguas cuyo nombre es conocido por otras informaciones y también constataremos los apelativos cuyo origen se puede rastrear en el nombre de ciudades actuales, cuando la relación entre ambos tenga un suficiente grado de probabilidad.

Es sabido que la divinidad indígena masculina que cumple estos requisitos con mayor asiduidad es Bandua. En primer lugar, muchos de sus epítetos nos remiten a topónimos con sufijación en -briga:

$\left.1 .^{\circ}\right)$ Etobrico. Conocido por una inscripción hallada en Alenquer (Lisboa), pero cuya procedencia original se desconoce ${ }^{12}$.

2. $\left.{ }^{\circ}\right)$ Brialeacui. En dos inscripciones de Orjais (Covilhã, Castelo Branco) ${ }^{13}$.

3. ) Isibraiegui. Conocido por varias inscripciones de Bemposta (Penamacor, Castelo Branco) y derivaría, según Almeida, del nombre primitivo de la localidad ${ }^{14}$.

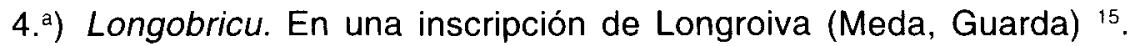
Langobriga se situaba según el Itinerario de Antonino entre Aeminium (Coimbra) y Cale (Porto) pero, como afirmaba Vasconcelos, ello no implica que debiera estar en la línea recta Olisipo - Aeminium-Cale, sino que podría ubicarse más al interior, como el ara dedicada a Bandua parece confirmar. En documentos medievales la aldea aparece como Longrobia y Longrovia ${ }^{16}$. Según Curado, lo más probable es que el topónimo al que alude el epíteto hubiera sufrido la evolución Longobriga - Longobria- Longroiva ${ }^{17}$.

LONGNON, 1968, 40-42; RIVET et al., 1979, 277-278.

2 Ferreira y Almeida, 1976, 139-142; Encarnaçäo, 1976, 142-146.

13 ALmeIDA, 1965, págs. 24-25; EnCARNAÇĀo, 1975, 125-126. De la segunda ara da noticia ENCARNAÇĀo $(1987,19)$.

14 Almeida, 1965, 19-22, n. ${ }^{\circ}$ y pág. 31; Albertos y Bento, 1975, 1208. La segunda ara la publicó ALMEIDA $\left(1965,22-23 \mathrm{n} .^{\circ}\right.$ 2). Posiblemente, también se habrian dedicado a este dios dos aras más, una hailada en el mismo lugar, Bemposta, y otra procedente de Freixo de Numão (Vila Nova de Foz Côa, Guarda), publicadas por LEITAAO y $0(1980,632-633)$ y por COIXÁO y ENCARNAAÇÃO $\left(1997,4,{ }^{\circ}{ }^{\circ} 3\right)$.

15 CuRAdo, $1985, n .^{\circ} 44$.

16 VASCONCELOS, 1905, 34 con nota 3. Alarcão $(1990,27)$ identifica Langobriga con Cabeço de Vouga (Agueda, Aveiro) por lo que, según él, no correspondería con el lugar del hallazgo de esta pieza.

17 Curado, $1985, n .^{\circ} 44$. 
5. ${ }^{\circ}$ Virubrico o Verubrico. En un ara de Retorta (Laza, Orense) ${ }^{18}$. Existen algunas dudas sobre las últimas letras del teónimo y las primeras del epíteto, pero parece clara la vinculación del dios a un enclave fortificado.

$\left.6^{\circ}{ }^{\circ}\right)$ Veigebreaego. Procedente de Rairiz de Veiga (Orense) ${ }^{19}$. Según Rodríguez Colmenero, es muy probable una relación entre el epíteto y el nombre del lugar de procedencia de la pieza, por lo que habría que admitir que el dios era el "protector do Castro da Veiga" ${ }^{20}$.

7. $\left.{ }^{\circ}\right)$ Lansbricae. De Santa Eugenia de Eirás (S. Amaro, Orense) ${ }^{21}$. Como vimos arriba, el ara se halló cerca de dos castros, uno de los cuales estaba en San Ciprián de Las. Según Rivas, la relación entre este topónimo y el epíteto Lansbricae quedaría reforzada a partir de un documento de 1458 conservado en el Archivo de la Catedral de Orense, donde se alude a ese enclave como Laans ${ }^{22}$.

8. ${ }^{\circ}$ Saisabro. De Maranhão (Avís, Evora) ${ }^{23}$.

9..$^{\circ}$ Malunrico? De procedencia desconocida, actualmente en el Museo de Badajoz. Según Ramírez Sádaba, la lectura más apropiada sería Malun<b>rico habiéndose omitido la letra $b$ por error del lapicida, lo que resolvería la complejidad fonética del epíteto ${ }^{24}$.

Por otra parte, también conocemos el nombre de otros núcleos urbanos antiguos que, aunque no posean los mencionados sufijos, dan lugar a sobrenombres de Bandua:

- Roudaeco. Este apelativo se refiere a un uicus Rouda conocido por una inscripción hallada en Casar de Cáceres ${ }^{25}$, que se ubicaría probablemente hacia la confluencia de los ríos Tozo y Almonte donde, como vimos arriba, se hallaron tres inscripciones dedicadas a Bandua Roudaeco. Además, cerca de Casar de Cáceres existe un lugar denominado Bando del Monte, posiblemente alusivo al teónimo Bandua, donde están las ermitas de $\mathrm{S}$. Benito y $\mathrm{S}$. Blas, lugares a los que llegan las

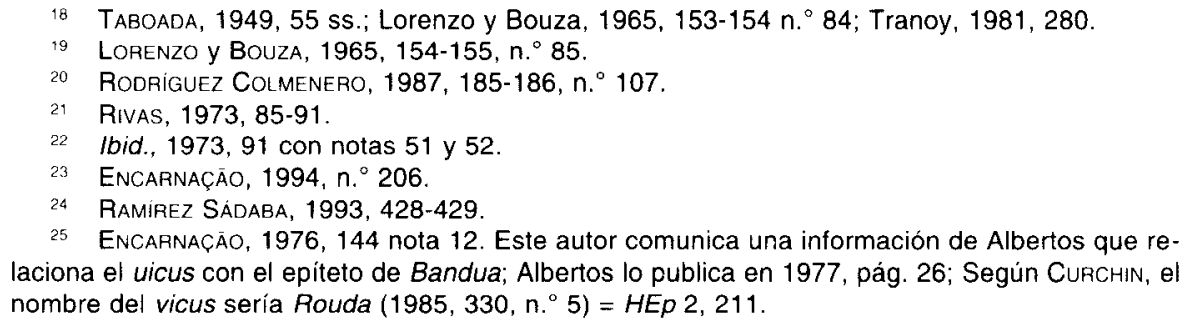
laciona el uicus con el epíteto de Bandua; Albertos lo publica en 1977, pág. 26; Según CuRCHIN, el nombre del vicus sería Rouda $\left(1985,330, \mathrm{n}^{\circ}{ }^{\circ}\right)=H E p 2,211$. 
principales romerias que parten del pueblo y que, por tanto, es el lugar de mayor tradición sacra de la zona.

- Oce... Procedente de Sul (S. Pedro do Sul, Viseu) ${ }^{26}$. J. d'Encarnação interpretó el epíteto como Ocel(ensi) o Ocel(aeco) ${ }^{27}$.

- Araugel(...). La inscripción se realizó en una pátera de plata cuya procedencia es desconocida. Hoy se encuentra en la colección Calzadilla de Badajoz ${ }^{28}$. No obstante, según Albertos y Alarcão, el epíteto podría referirse a un castellum Araocelum ubicado en el área del actual Viseu, a partir de la inscripción hallada en S. Cosmado (Mangualde) donde se menciona unos castellani Araocelenses ${ }^{29}$.

A los nueve epítetos alusivos a ciudades con sufijo -briga arriba mencionados hemos de sumar, por tanto, los tres últimos que aluden a comunidades urbanas lusitanas. Junto a estos doce epítetos, conocemos otros diez bastante probables cuyo significado desconocemos: Apolosego con sus variantes, Arbariaico, Bolecco, Cadiego, Oilienaico, Picio, Tatibeaicui, Tueraeo, Velugo Toiraeco y Vortiaecio con todas sus variantes. Por tanto, de un total de 22 epítetos del dios Bandua, el 54,5\% derivan de nombres de localidades antiguas, proporción que se reduce al $40 \%$ si no incluimos los tres apelativos de más dudosa vinculación a núcleos de población: Oce..., Saisabro y Malunrico. Es una cifra, a nuestro juicio, altamente significativa y susceptible de ser sometida a comparaciones con otros ámbitos. Del resto nada fiable podemos afirmar en cuanto a su tipificación.

Finalmente, hemos de considerar como importante la evidencia de que, exceptuando el epíteto Vortiaecio y sus variantes, que aparece documentado por un amplio territorio, y uno de los testimonios de Roudaeco, que se halló desplazado del resto, cada uno de los apelativos de Bandua se testimonia en una localidad concreta.

La consideración del resto de inscripciones donde aparecen epítetos como los citados nos aporta informaciones de gran interés. Los sobrenombres que derivan de topónimos en -briga son los siguientes:

- loueai Caielobrigoi. que aparece en una misma inscripción que Crougeai Magareaicoi, grabada sobre una gran roca en Lamas de Moledo

26 VASCONCELOS, 1905, 316.

27 EnCARNAÇĀO, 1987, 20.

28 BLANCO, 1959, 458 ss.

29 AlbeRTOS, 1985, 472; AlARCĀo, 1989, 307 y 309. La inscripción de los castellani se publicó en HAE 988 = ILER 5242. Según P. LE ROuX, se trataría del actual Viseu (1992-1993, 154). 
(Castro Daire, Viseu) ${ }^{30}$. Ambos epítetos aparecen en topónimos locales: Magareaicoi se vincularía al Castro de Maga, situado en un cerro frente a Lamas de Moledo; Caielobrigoi habría dado lugar a Cela, población vecina de Lamas ${ }^{31}$.

- Soe Meobrigoe. De procedencia desconocida, pero probablemente hallada en la provincia de La Coruña ${ }^{32}$.

- Genio Conimbricae. Procedente del recinto de Conimbriga ${ }^{33}$.

- Genio Tongobrigensium. Aparecido en Freixo (Marco de Canavezes, Porto) ${ }^{34}$. El epíteto se refiere, probablemente, al gran castro en el que se halló la inscripción. Su nombre sería Tongobriga, llamada en el Ravenate Tonobriga, o Tuntobriga por Ptolomeo ${ }^{35}$.

- Tameobrigo (Varzea do Douro, Marco de Canavezes) ${ }^{36}$. La inscripción apareció en la confluencia entre los ríos Douro y Tâmega; la raíz Tam-podría indicar una relación entre esta divinidad y el río Tâmega. La naturaleza de esta relación ha sido debatida por los distintos investigadores que han estudiado este testimonio. Para Blázquez se trataba de una divinidad acuática vinculada al río ${ }^{37}$, mientras que Encarnação pensaba que se trataba del propio río divinizado ${ }^{38}$. Sin embargo, Tameobrigo posee el sufijo característico de los epítetos tópicos de divinidades indígenas relacionados con enclaves fortificados.

- (...)a Brigo? (Delaes, Vila Nova de Famalicão). A la inscripción le faltaría una primera línea donde se ubicaría quizá parte del teónimo, cuya última letra sería la a de la primera línea conservada. Brigo sería, así, el epíteto de una divinidad cuyo nombre desconocemos.

Finalmente, también serían derivados de topónimos de poblaciones los siguientes:

30 CIL II 416 y pág. 695. loueai podria ser una variante dialectal de Júpiter ( $A E$ 1989, 382) Sin embargo, la mayoría de los autores no opinan en este sentido.

31 VAZ, 1993, 238.

32 LE ROUX Y TRANOY, 1973, 225-226

33 ALARCÁO et al., 1969, 217-218; ETIENNE et al., 1976, 24-25 n. ${ }^{\circ} 6$.

34 CIL II 5564; CARDOZO, 1935, n. ${ }^{\circ} 35$; ENCARNAÇĀO, 1975, 195-197; GaRCIA, 1991, n. 205

35 VASCONCELOS, 1905, 196 con nota 3. Por otra parte, en Brozas (Cáceres) se halló un altar votivo dedicado a Júpiter por unos uicani Tongobrigeses, sin que sepamos con seguridad si se trata de otro enclave con el mismo nombre o si alude al mismo que el epiteto del Genio (CURCHIN, 1985, 330 $\left.n .^{\circ} 6\right)$. En cuanto a la ubicación del uicus en Brozas, lo que parece más probable, uid. infra.

36 CIL II 2377; Vasconcelos, 1905, 319-321; Cardozo, 1935, n. ${ }^{\circ}$ 14; ILER 931; Garcia, 1991, n. 193.

37 BlAzQuez, 1962, 190. Una opinión parecida mantiene Tranoy, para quien Tameobrigus sería "le maitre» del río $(1981,277)$.

38 EnCARNAÇÄO, $1975,276-280$. 
- Marti Tarbuceli. Aparecido en un ara procedente de Montariol (S. Victor, Braga) ${ }^{39}$. Lo incluimos aquí a partir de una inscripción hallada en Pastoria (Chaves), en la que se cita un castellum Tarbu... ${ }^{40}$ sobre el que, probablemente, este Marte indígena ejercería su influencia. Según Albertos, la variante -ucelum en composición de topónimos consta en Albucela, Araugelensis, Balatucelum o Arcucelum, por lo que el epíteto de Marte derivaría de Tarbucelum ${ }^{41}$.

- Durbedico. Altar procedente de Ronfe (Guimarães, Braga) ${ }^{42}$. Sobre el significado de este epíteto se han planteado diversas opiniones, en el sentido de que es una divinidad de las aguas ${ }^{43}$. Sin embargo, la existencia de una inscripción que cita un castellum Durbede que estaría ubicado en el área de Braga es determinante ${ }^{44}$. No sabemos el nombre de la divinidad a la que se refería la inscripción.

- Igaedo. Lo conocemos por una inscripción hallada en una dependencia agrícola existente detrás de la capilla de N. ${ }^{a}$ Sra. de Almortão (Idanha-a-Nova, Castelo Branco) ${ }^{45}$, cerca de Idanha-a-Velha, lugar donde en época romana estaba la capital de la ciuitas Igaeditanorum. Este apelativo haría referencia, por tanto, a esa gens o a la propia capital que, según Vasconcelos, pudo llamarse Igaedi o Igaedium, que posteriormente derivó a Igaeditania como aparece en monedas de época visigoda ${ }^{46}$. Por tanto, es difícil discernir entre una adscripción étnica o ciudadana de este apelativo.

- Carneo Calanticensi. Este epíteto es conocido por una inscripción desaparecida y aludiría, según Encarnação, a una población que se denominaría Calantum, Calanta, Calantica o Calantia ${ }^{47}$.

- Genio Laquinie(n)si (S. Miguel de Caldas de Vizela, Guimarães) ${ }^{48}$. La mayoría de los autores que han estudiado la pieza inciden en el carác-

\footnotetext{
39 Tranoy, 1981, 304; Santos et al., 1983, 192; Tranoy, 1984, 446-447

40 Albertos, $1975,33, n .{ }^{\circ} 18$. Sobre el carácter de la " $C$ " invertida como símbolo de un castellum, uid. pág. 65-66.

41 Albertos, 1985, 472

42 Hallada en 1881 en la torre de la iglesia de Ronfe (SARMEnTO, 19331, $177 \mathrm{n}^{\circ} 8$ ).

43 Vasconcelos, 1905, 329-331; BLÁZQUez, 1962, 174; ENCARNAÇĀo, 1970, 228-230 n. ${ }^{\circ} 8$; Encarnaçäo, 1975, 179; TranoY, 1981, 274-275.

44 EnCARNAÇÃo, 1984, 187-188. Para P. LE Roux, el nombre del enclave sería Durbeda o Durbedum (1992-93, 154 n. $\left.{ }^{\circ} 3\right)$.

45 ALMEIDA, 1964, 65-73; ILER 5995; ENCARNAÇĀO, 1975, 199-200.

46 VASCONCELOS, 1905,32 con nota 3.

47 EnCARNAÇÃo, $1984,489, \mathrm{n}^{\circ}{ }^{\circ} 410$. Pudieron haberse dedicado dos altares más a este dios, pero el primero de ellos está también desaparecido y el segundo es bastante dudoso (ibid., 490$491, n .{ }^{\circ} 411$ y 412 ).
} 
ter tópico o étnico del epíteto, que podría aludir a una ciudad o territorio de nombre Laquinia o Laquinium ${ }^{49}$. En Caldas de Vizela hubo posiblemente un uicus llamado Oculis, que en el año 1014 aparece como Occulis Calidarum ${ }^{50}$ que, aparentemente, no tendría relación con el apelativo de la divinidad. Si este dato se confirma, aumentarían las dudas sobre el significado del epíteto.

- Conso S[...]ensi (S. Pedro de Trones, Puente de Domingo Flórez, León) ${ }^{51}$. Es una dedicación a Coso y un ejemplo de la vinculación de este dios a un enclave, puesto que el adjetivo deriva de un topónimo.

- Coso Vacoaico (lugar indeterminado del distrito de Viseu) ${ }^{52}$. Posiblemente el epíteto alude al oppidum Vacca que se cita en Plinio. Este enclave estaría cerca del lugar donde se halló la inscripción de Coso y también del río Vouga, pues llevaba su mismo nombre ${ }^{53}$. No obstante, la relación del topónimo y el apelativo de Coso no es del todo segura.

- Coso Neneoeco. Conocido por dos inscripciones halladas en el concelho de Santo Tirso (Porto) ${ }^{54}$. Alarcão sugería la posible relación de este epíteto con el nombre de una localidad de la zona: Nine ${ }^{55}$, aunque esta vinculación no se puede asegurar por el momento.

- Arantio Ocelaeco. Esta ofrenda procedente de Ferro (Covilhã, Castelo Branco) ${ }^{56}$, se hace al dios junto a Arantia Ocelaeca. Para Albertos, Ocelum sería el lugar que protegerían ambos dioses, pero sería distinto a la ciuitas de los Ocelenses que mencionan Ptolomeo y Plinio y al Ocelum de los Vettones, aunque podría ser también este último enclave ${ }^{57}$.

- Lari Ocaelaego. En esta inscripción, hallada en Santa Magdalena de Paradiña (Sarreaus, Orense), consta otro epíteto del mismo tipo que el anterior, alusivo a un topónimo Ocelum ${ }^{58}$.

Además de las inscripciones que citamos, Albertos incluyó otras en las que los epítetos se relacionaban con núcleos de población. Se trata de las

48 CIL II 2405; CARDOZO, 1935, n. 36; ILER 658; GARCIA, 1991, n. 206.

49 VASCONCELOS, 1905, 195; en el mismo sentido, BLAZQUEZ $(1962,135)$ y ENCARNAÇÄo $(1975$, 191-192)

50 Alarcão, $1988,17 \mathrm{n} \cdot{ }^{\circ} 315$.

51 Garcia Martinez, 1998, 325-331.

52 VAZ, $1989, \mathrm{n}^{\circ} 140$.

53 AlLARCĀO, 1974, 91; Vaz, 1989, n. ${ }^{\circ} 140$

54 BLÁZQUEZ, 1962, 120-122; ENCARNAÇĀO, 1975, 164-169.

55 Alarcāo, 1974, 171; Garcia, 1991, n. ${ }^{\circ} 50$.

56 LEITÃO, 1981, 56-58.

57 Albertos, 1985, 472-473.

58 Rodriguez González, 1990-1991, 204-209. 
referentes a Arentio Cronisensi, Bandua Ituicie(n)si, Laribus Cusicelensibus, dis deabus Nobranininsibus ${ }^{59}$ y, en un trabajo posterior, añadió a estos otros apelativos que tenian relación con topónimos actuales: Segidiaeco, Cariociego, Paraliomego, Paramaeco, Barciaeco, Tabudico, Circeiebaeco y Tarmucenbaecis ${ }^{60}$. No obstante, las lecturas de algunos epítetos del primer grupo no son lo suficientemente fiables para permitirnos su utilización con fines ulteriores; en cuanto al segundo grupo, como ya Albertos anunciaba, no es seguro que esos epítetos se identifiquen con los nombres de topónimos actuales que cita.

En resumen, además de los doce testimonios referidos a Bandua, disponemos de tres dedicaciones a Genios indígenas con epítetos derivados de topónimos, tres a Coso ${ }^{61}$ y una a Marte, los Lares, loueai (que quizá corresponda a Júpiter), Crougeai, Soe, Carneo y Arentio. Finalmente tenemos altares ofrecidos a dioses cuyo nombre no conocemos, pero sí sus epítetos: Tameobrigo, Brigo, Durbedico e Igaedo.

Hemos expuesto los datos que muestran algo ya conocido por los investigadores, que en el territorio lusitano-galaico Bandua es la divinidad indígena a la que con mayor frecuencia se cita junto a epítetos referentes a uici, pagi o castella, por lo que se puede concluir que tenía una muy especial vinculación con comunidades indígenas de segundo rango. A la gran proporción de invocaciones a Bandua con epítetos que lo caracterizan como divinidad vinculada de diversas poblaciones ${ }^{62}$ se une, por el contrario, la ausencia total de apelativos de este dios referentes a grupos familiares, clánicos o tribales.

Es cierto que no conocemos el significado de un buen número de sobrenombres de Bandua y podrian haber entre ellos apelativos gentilicios, pero este punto no se puede confirmar con los datos actuales ${ }^{63}$. Además, hemos de considerar que otras divinidades de las que conocemos menos testimonios se acompañan de sobrenombres alusivos a grupos familiares. Podemos citar algunos ejemplos: Araco Arantoniceo relacionado con el antropónimo Arantonius, Arentio Tanginiciaeco vinculado a Tanginus, los Lares Gapeticorum gentilitatis y los Lubanci Douilonicorum ${ }^{64}$, los Lares Cerenaecis, con posible vinculación a Cereaecius; Lugubo Arquienobo y

AlBertos, 1975,60

60 Albertos, 1977, 25.

61 De ellas una se vincula claramente, por el epiteto, a un topónimo y en las otras dos la relación no es segura. Por otra parte, la ofrenda a Soe Meobrigoe podría pertenecer también a esta divinidad.

62 Albertos, 1977, 21.

63 De HOZ, 1986, 41.

64 Albertos, 1975, 51. 
Lucoubo Arquieno, relacionados con el antropónimo Arquius 65 o Mentouiaco, epíteto cuyo teónimo desconocemos que guarda relación con el gentilicio Mentouiequm ${ }^{66}$.

J. De Hoz ya había advertido que Bandua era una divinidad protectora y tutelar, ligada a "comunidades humanas", pero consideraba que no era una divinidad personal, con perfiles bien definidos, sino con un carácter genérico ${ }^{67}$. Además, este investigador planteaba que los Genios o Lares que aparecen en singular en las inscripciones pudieran tener características similares a las de Bandua ${ }^{68}$.

Hemos visto arriba que tres de los ejemplos hispanos donde aparecen epítetos relativos a comunidades urbanas son dedicaciones a Genios lo que está, en principio, de acuerdo con las hipótesis de J. De Hoz respecto a una cierta identificación entre ambas divinidades. Pero, si esta idea es correcta, ¿qué información nos aporta para establecer el carácter de Bandua? ¿Qué características tenían los Genios romanos que parecían similares a una divinidad que ejercía su tutela sobre comunidades indígenas?

Sabemos que el culto al Genio, durante el alto imperio, estaba lejos del casi exclusivo perfil familiar que tenía durante los primeros tiempos de la República. En todo el mundo romano hay testimonios de Genios que ejercían protección sobre poblaciones, demarcaciones administrativas, collegia, etc. ${ }^{69}$. No obstante, uno de los ámbitos donde más rastros han quedado del culto a Genios ha sido en el militar, donde aparece como protector de diversas unidades, instalaciones o dioses de carácter guerrero ${ }^{70}$. Por otra parte, determinados Genios eran entidades que protegian un gran número de localidades en todo el mundo romano.

Es, por tanto, lógico que el dios al que se encomendaba la protección de las comunidades indígenas durante el imperio romano y que antes de la conquista tutelaría a la unidad política también en las lides militares, fuera asimilado en determinadas ocasiones al Genio romano que tenía un contenido religioso similar.

La repetición de este tipo de sobrenombres calificando a una misma divinidad indígena en la Hispania indoeuropea nos lleva a plantear si algunas

5 TrANOY, $1981,378$.

AlbERTOS, $1975,17, \mathrm{n} .^{\circ} 166$

DE HOz, 1986, 41.

Ibid. loc.cit.

IGLESIAS, 1986, 129-132.

Speidel y Dimitrova-Milceva, 1978, 1543-1551. 
divinidades adoradas en otras regiones del Occidente del imperio romano eran, con mayor frecuencia respecto al resto, aludidas con epítetos vinculables a localidades. Nos parece necesario realizar esta pesquisa para averiguar si los datos nos ofrecen algunos términos útiles de comparación con los datos obtenidos en la Península Ibérica.

\section{DIVINIDADES CON EPITETOS VINCULABLES A POBLACIONES EN LAS GALIAS Y BRITANIA}

Como ya había observado Reinach, Marte es una de las divinidades que posee más apelativos tópicos de raíz indígena en todo el Occidente romano. La etimología de algunos de ellos nos remonta a nombres de poblaciones.

- Albarino ${ }^{71}$. Es plausible que aludiera al nombre antiguo de la población de Aubaroux, lugar donde se halló la inscripción ${ }^{72}$.

- Albiorigi. Según Olmsted significaría "Rey de los Albionenses» O "Rey del Mundo", aunque la inscripción dedicada a la diosa Albiorica induce a pensar que el epíteto denota el carácter local del dios. De este modo, estos nombres podrían relacionarse con el pagus Albionensis en la región de Basses-Alpes ${ }^{73}$.

- Boluinno ${ }^{74}$. La inscripción procede de Bouhy (Nièvre), cuyo nombre antiguo daría lugar a este epíteto ${ }^{75}$. Sin embargo, Thévenot plantea que las formas más antiguas del topónimo entre los siglos vII y $x$, Baugiacus y Balgiacus postulan un tema primitivo Balbiacus que no tendría una clara relación con Bouhy ${ }^{76}$.

- Budenico 77 . Marte sería una divinidad protectora para los Budenicenses, que son nombrados en otra inscripción procedente del mismo lugar, Collias (Gard) ${ }^{78}$.

1 CIL XII 1157.

HeichelHeIM, 1930, 1941; Lambrechts, 1942, 131.

HeICHELHEIM, 1930, 1941; OLMSTED, 1994, 345-346; BARRUOL opinaba que su significado podía ser el de "rey de los Alpes", aunque dada la situación de los testimonios, es una tesis cuestionable (1963, 356 ss.).

CIL II 2899 y 2532.

OLMSTED, 1994, 346.

THÉVENOT, 1955, 33.

CIL XII 2973.

Heichelheim, 1930, 1942-43; ThéVenot, 1955, 88 n. 1. Según Fleuriot, el galo "budenikos significaría "qui a rapport avec l'armée" $(1982,121)$. Sin embargo, como en los otros casos, el apelativo de Marte indica, en primer lugar, una vinculación con el citado uicus. 
- Buxeno. Este epíteto relaciona a Marte con el Campus Buxonus (Buisson, cerca de Vellerón, Vaucluse) ${ }^{79}$.

- Cemenelo. Alusivo a la comunidad de Cemenelum (Cimiez, AlpesMaritimes) ${ }^{80}$.

- Cuntino. La inscripción, hallada cerca de Contes (Alpes-Maritimes), está dedicada a Marte [S]egomoni Cuntino ${ }^{81}$ en la que, además, el dedicante es el propio uicus Cuntinus, por lo que se refuerza desde todos los puntos de vista la adscripción de Marte a la protección de los habitantes del citado uicus.

- Dunati. Según Olmsted, significaría "(Protector) de la ciudad fortificada". Para este autor, dicho campo semántico lo acercaría a otro epíteto como Toutatis ("protector de la tribu»). El céltico dunon ("ciudad fortificada") se podría interpretar como el lugar central de la tribu, por lo que el dios funcionaría como el "protector del centro tribal" ${ }^{82}$.

- Giarino. Según Olmsted, deriva del nombre de una localidad de etimología no celta ${ }^{83}$.

- Intarabo. En opinión de Jullian, podría tener relación con el nombre antiguo de Entrains (Nièvre), Intaranum, pero la distancia entre este lugar y el territorio donde aparecen las dedicaciones a Marti Intarabo, en torno a Trier (Rheinland-Pfälz) cuestionan en buena medida esta tesis ${ }^{84}$.

- Lacauo. Deriva, a juicio de Olmsted, del nombre de una localidad de etimología no celta ${ }^{85}$.

- Mulloni. Es posible su relación con un nombre de localidad que tendría el significado de "colina» ${ }^{86}$.

- Randosati. Aludiría al enclave de Randanum, hoy Randan, cerca de Rionn (Puy-de-Dôme) ${ }^{87}$.

- Rudiano. Se ha establecido una relación entre este apelativo y el nombre del pagus Royannensis, atestiguado en el siglo x y, en la actualidad,

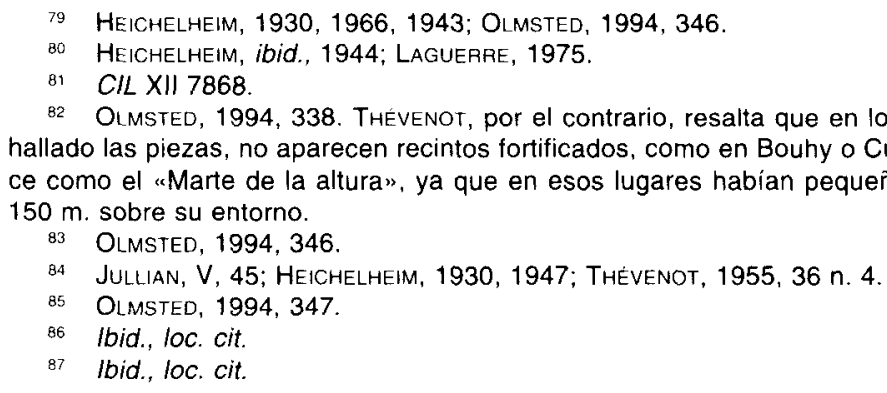
$150 \mathrm{~m}$. sobre su entorno.

83 OLMSTED, 1994, 346.

84 Jullian, V, 45; Heichelheim, 1930, 1947; ThéVenot, 1955, 36 n. 4.

85 Olmsted, 1994, 347

86 Ibid., loc. cit.

87 Ibid., loc. cit.

32 Olmsted, 1994, 338. ThÉVEnOt, por el contrario, resalta que en los lugares donde se han hallado las piezas, no aparecen recintos fortificados, como en Bouhy o Culoz, por lo que lo traduce como el "Marte de la altura", ya que en esos lugares habian pequeñas elevaciones de 80 y 
Royans ${ }^{88}$. El nombre del pagus podría haber sido citado en la antigüedad como Rudianensis. Thévenot, en cambio, plantea muchas resenvas a esta tesis, puesto que las dedicaciones donde consta el epíteto se extienden por un gran territorio y, en algunos casos, se alejan bastante de la ubicación de Royans ${ }^{89}$.

- Vesontio ${ }^{90}$. Relativo a Vesontio, nombre antiguo del actual Besançon, donde se halló la inscripción ${ }^{91}$.

- Vicinno ${ }^{92}$. Según Olmsted es probable que su significado fuera «el luchador" o "el guerrero" ${ }^{93}$. Sin embargo, podría derivar del nombre antiguo de la Vilaine que es Vicinonia en Gregorio de Tours ${ }^{94}$. En cualquier caso, Thévenot cree que Marte Vicinno protegería un pagus, puesto de el altar se hace en honor del pagus Carnutenus ${ }^{95}$.

- Vintio ${ }^{96}$. Aunque aparece vinculado a Polux en dos inscripciones, sería el mismo que el Mars Vintius de Vence (Alpes-Maritimes) ${ }^{97}$, donde la relación entre epíteto y topónimo es evidente. El hecho de que el culto a Marte indígena tenía una objetivación local se plasma en la citada inscripción de Vence, puesto que es realizada por un individuo que se declara incola de Cemenelum, lugar donde se adoraba a Mars Cemenelus ${ }^{98}$. Sin embargo, cuando este hombre hace la dedicación en Vence, cita a Marte con su epíteto local, Vintius y no con el de su ciudad de origen.

- Vorocio. Derivado de Vorocium, hoy Vouroux (Allier) ${ }^{99}$.

Otras inscripciones podrían vincular también a Marte a determinadas poblaciones. Un ejemplo, como mostraba Thévenot, sería la dedicación a Mars y a Vasio procedente de Vaison (Vaucluse), es decir a Marte junto a una divinidad que sería la protectora de la comunidad, lo que otorgaría el mismo papel al dios ${ }^{100}$.

\footnotetext{
HEICHELHEIM, 1930, 1954.

89 ThÉVENOT, 1955, 104 SS. Para OLMSTED, este epíteto sería un adjetivo que significaría "fuerte" o «robusto" $(1994,344-345)$.

CIL XIII 5368.

THEVENOT, 1955, 56 n. 2

CIL XIII 3150.

OLMSTED, 1994, 345.

Hist. Fr. 5, 26.

THÉVENOT, 1955, 118.

CIL XII 2561 y 2562.

CIL XII 3. HeICHELHEIM, 1930, 1957; ThEVENOT, 1955, 90

Vid. SUPRa. BENOIT, 1977, 53.

HEICHELHEIM, 1930, 1957.

CIL XII 1301 y, probablemente, 1336; ThEVENot, 1955, 103 n. 5.
} 
Los epítetos referentes a localidades acompañaban también a otras divinidades masculinas pero, a la luz de los datos, en mucha menor medida que a Marte. Como es bien conocido, hay ejemplos de enclaves tutelados por Genios y, paradójicamente, hay pocos datos más referentes, únicamente a otros dos dioses: Mercurio y Hércules. En cuanto a Mercurio, conocemos tres sobrenombres de este tipo: Canetonnesi, citado en un altar de Bernay (Eure), que aludiría a la localidad de Canetonnum (Le Villeret, Eure) ${ }^{101}$; Magniaco ${ }^{102}$, posiblemente local por haber sido hallada la inscripción en Magnieu ${ }^{103}$ y Alauno, procedente de Aulun ${ }^{104}$ de cuyo nombre antiguo derivaría el apelativo ${ }^{105}$. Respecto a Hércules, sólo conocemos un testimonio de este carácter: Deusoniensi, que aludiría a la población de Deusonia ${ }^{106}$.

La primaria definición del carácter religioso de Bandua que establecimos arriba adquiere unos perfiles más concretos, por tanto, si tenemos en cuenta los datos referentes a las provincias galas: aquí, donde una gran parte de los teónimos indigenas desaparecieron bajo sus paralelos romanos, la divinidad más relacionada mediante sus epítetos con núcleos de población es Marte. Además, los apelativos indigenas de este tipo suman el $24 \%$ del número total de los referidos a este dios ${ }^{107}$, lo que supone una proporción bastante considerable que está lejos de los datos conocidos respecto al resto de divinidades.

Ahora bien, si Bandua o divinidades indígenas equivalentes con otro nombre habían tenido en algún período la significación política que afirmamos, los casos de interpretatio no se darían únicamente en un ámbito privado. Por tanto, nos queda explicar cómo se produce la interacción de cultos, indígenas y romanos, en el seno de las comunidades locales lusitano-galaicas.

\section{INTERACCIÓN ENTRE LOS DIOSES TUTELARES INDIGENAS $Y$ ROMANOS}

Etienne, en un estudio referido a las Galias, afirmaba que los pagani rendían culto en el período prerromano a una divinidad protectora del pagus que después de la conquista romana sería designada como Genius.

\footnotetext{
101 Heichelheim, 1931, 991; Olmsted, 1994, 346.

102 CIL XII 2373.

103 LAMBRECHTS, 1942,124 n. 8.

104 CIL XII 1517.

105 HeICHELHEIM, 1931, 989; LAMBRECHTS, 1942, 124 n. 3.

106 Olmsted, 1994, 403-404.
} 
Así, la personalidad de estos pagi, que pierden con la conquista gran parte de su significado político, sería respetada mediante la exaltación religiosa de su Genius ${ }^{108}$. Según Etienne, en los templos del culto imperial tendrían su lugar los numina pagorum, erigiéndose de este modo la capital de la ciuitas galo-romana en el centro federal donde los distintos grupos sociales tendrían su lugar en el seno del culto imperial ${ }^{109}$. Así, el culto al emperador desarrollado en la capital de la ciuitas tendría un efecto centralizador y unificador que colaboraría a la reducción y eliminación de las fuerzas centrífugas que podían suponer los pagi, con sus sólidas tradiciones religiosas.

La gran importancia de los Genios de uici o pagi como divinidades tutelares del conjunto de una comunidad frente al resto de enclaves es bastante patente en la inscripción de Hasparren hecha en honor del aquitano Vero, que había logrado mediante una legación al emperador la segregación de los Nouem populi del resto de los Galos: "Vero, flamen, duunuiro, cuestor y magistrado de su pagus, tuvo el honor de participar en la delegación enviada ante el Augusto y que obtuvo la separación de los Nueve Pueblos del resto de los galos. De regreso a Roma dedicó esta ara al Genio del pagus" ${ }^{110}$. El hecho de que el ara conmemorativa de semejante misión fuera dedicada al Genio del pagus deja pocas dudas sobre el carácter político que los magistrados de estas comunidades $y$, por consiguiente, el conjunto de sus habitantes otorgaban a esta divinidad.

Por otra parte, Hatt observaba que en las dedicaciones galas a Marte con epítetos indígenas participaron en gran medida altos magistrados de rango consular, oficiales ecuestres y un buen número de flamines además de numerosas colectividades. Desde este punto de partida, Hatt consideraba que la fuerte propaganda a favor de los "Martes indígenas" establecida por las clases dirigentes a partir de finales del siglo I d.C. era, de modo similar al planteado por Etienne para los Genios locales, una con-

107 Esta proporción es bastante aproximada, si bien hemos de tener en cuenta que la etimo. logía de algunos de los epítetos está sometida a discusión. No obstante. Reinach ya habia observado a principios de siglo que el número de epítetos tópicos de Marte era muy superior al de otros dioses como Mercurio, cuyos sobrenombres eran de un carácter más general (1913, 179).

108 ETIENNE, 1993, 172-173. Este autor cita el Genio del pagus Tigurinus, perteneciente a la ciuitas Heluetiorum y, en la capital de esta comunidad, Auenticum, la dedicación a la Dea Auentia y al Genio de los incolae.

109 ETIENNE, 1993, 174.

t10 SAYAS, 1990, 176. CIL XIII 412: Flamen item dumuir quaestor pagiq. magister/Verus ad Augustum legato munere functus / pro nouem optinuit populis seiungere Gallos / Urbe redux Genio pagi hanc dedicat aram. 
traposición tendente a neutralizar las tendencias a la resistencia nacional de la religión druídica ${ }^{111}$.

Los testimonios de epítetos locales que citamos arriba nos permitian observar cómo la romanización de los teónimos indígenas correspondientes a divinidades tutelares, principalmente Marte, llegó en las Galias hasta los enclaves menores, uici y pagi, de manera muy intensa. Hay que insistir en que este dios es, según los datos actuales, el que más apelativos de este tipo tiene registrados en las provincias galas.

La información referente a la Hispania occidental varía, sin embargo, en algunos aspectos respecto a los datos alusivos a las Galias. En primer lugar, el mayor número de sobrenombres derivados de núcleos de población acompaña a un teónimo indígena: Bandua. Se detecta, por tanto, una mayor capacidad de resistencia por parte de las divinidades tutelares lusitano-galaicas a los cambios derivados del impacto cultural de Roma. No obstante, esta resistencia no se dio en todos los lugares por igual.

En este sentido, es destacable que en tres de los altares dedicados a Genios con apelativos de localidades en el territorio que estudiamos, esta divinidad tutelara Conimbriga, Tongobriga y Turgalium que, aunque tenían un gran componente indígena en su población, eran comunidades de primer orden desde el punto de vista de su extensión y carácter administrativo estando, además, altamente romanizadas ${ }^{112}$.

Esta afirmación vale respecto a otros Genios relacionados con poblaciones. En este sentido, conocemos un testimonio de un Genius municipii procedente, posiblemente, del municipio que se ubicaría en Bobadela ${ }^{113} \mathrm{y}$ otro hallado en Sellium (Tomar), importante municipio situado en la vía que unía Olisipo con Bracara Augusta ${ }^{114}$. Finalmente, tenemos otra dedicación a un Genius oppidi de S. Salvador de Aramenha (Marvão, Portalegre), donde se situaba la capital de la ciuitas Ammaiensis ${ }^{115}$. Nos consta, sin embargo, una dedicación a un Genius castelli, aparecida en la iglesia parroquial de Cores (Ponteceso, La Coruña) ${ }^{116}$ que probablemente

111 HATT, 1989, 156-157.

112 Aunque en el caso de Tongobriga, la afirmación no es del todo segura, uid. ALARCäo, $1988,28 n .^{\circ} 1 / 482$ y DIAS, 1993-1994, 111.

113 AlARCẢo $(1974,167)$ cree que fue hallada en Bobadela, desde donde se trasladó a la capilla de S. Sebastiāo de Midões (Tábua); Garcia, 1991, n. ${ }^{\circ} 258$; MANTAS, 1993, 229-230.

114 Alarcảo, 1974, 167; id., 1990, 26; Tranoy, 1990, 14.

115 Alarcáo, 1974, 167; id., 1988, II, 148; id., 1990, 23. Para AlaRCÁO, habría sido Claudio quien habría otorgado a este enclave la capitalidad de la ciuitas siendo él o su sucesor los que la habrian otorgado la municipalidad.

116 Pereira, 1994, 177, n. ${ }^{\circ} 67$ 
correspondería al castellum Aviliobris citado en otra inscripción procedente del mismo lugar ${ }^{117}$. En resumen, casi todas las menciones de Genios locales hispanos aparecen vinculados a comunidades de elevado rango. En cambio, en las provincias galas y en Germania conocemos numerosas menciones a Genios de un determinado pagus o uicus ${ }^{118}$.

En cuanto a Marte indígena, ya planteamos en un trabajo anterior la hipótesis según la cual el territorio de culto de este dios era excluyente con respecto al ocupado por Bandua, lo que podía significar que ambas divinidades cumplían un mismo rol religioso ${ }^{119}$. El territorio donde se adoraba a Marte con apelativo indígena es, si lo comparamos con el de Bandua, testimonial. Posiblemente, la razón venga dada por las mismas causas que habíamos observado respecto a los Genios: al contrario que en las Galias, Marte no pudo cubrir en Hispania el papel encomendado a los dioses tutelares indigenas, al menos, en las comunidades menores sin relevancia administrativa o aisladas de los principales ramales viarios ${ }^{120}$. El único territorio donde se produce una excepción es, aproximadamente, el enmarcado por los ríos Miño y Lima, de donde proceden tres inscripciones dedicadas a Marte Carieco o Cairiogiego de cuyo epíteto no sabemos nada y, algo más al sur, el altar dedicado a Marte Tarbuceli ${ }^{121}$. El altar dedicado a Marte Boro, de Monsanto (Idanha-a-Nova), sería el único testimonio de Marte con epíteto indígena hallado al sur del río Duero.

Los escasos testimonios de Marte no están en núcleos muy romanizados y centrales desde el punto de vista administrativo. Ello podría significar que la interpretatio de esta divinidad no estuvo muy impulsada por el Estado romano como sí ocurrió con los Genios ciudadanos en el territorio lusitano-galaico o con Marte indígena en las Galias.

No obstante, tenemos algún ejemplo puntual del carácter que tomó la irrupción de estos cultos romanos en las principales comunidades lusitanas.

117 Pereira, 1982, 251; id. 1994, 175, n. 66

118 En cuanto a pagi: CIL XIII 5076: Genio pagi Tigorini; 4679: Genio pagi Deru(ensis); BINSFELD et al., 1988, 102, n. ${ }^{\circ} 195$ : Genio pagi Vilciatis; AE 1983, 728: Genio pagi Ac...; sin mencionar el nombre del pagus, CIL XIII 412 y 4680 . Respecto a vici, CIL XIII 3652, 5967, 6433, 7655 y 8838 .

119 Olivares, 1997,211 ss.

120 Tranoy ya propuso que los escasos testimonios de Marte indigena podrian tener relación con la gran cantidad de exvotos ofrecidos a Bandua o Cosus $(1981,315)$.

121 En el sitio de Pastoria, donde apareció el testimonio del castellum Tarbu, se hallaron también un miliario de Augusto y otro de Trajano en el que se contaba la milla $V$ a partir de Aquae Flauiae (Chaves). Por tanto, si el castellum se ubicaba en Pastoria, hemos de considerar que estaba muy cerca del citado municipio y en la vía de comunicación que unía a éste con Bracara Augusta (AlARCĀo, 1988, II, pág. $7 \mathrm{n},{ }^{\circ} 117$ ), lo que justificaría la romanización del teónimo. 
Nos lo brinda el evergeta Caius Cantius Modestinus, que construyó un templo a Marte en la capital de la ciuitas Igaeditanorum. Este individuo también había sufragado, entre otros, el templo dedicado al Genio de la ciudad que se ubicaría en Bobadela (Oliveira do Hospital, Coimbra) por lo que, en opinión de Mantas, las actuaciones de Modestinus reflejarían "o ambiente próprio das elites municipais das ciuitates lusitanas e o esforço que o referido grupo social desenvolveu no sentido de promover gradualmente a unidade política do território através de um ideal de solidariedade em torno dos cultos oficiais, quase sempre relacionados com o seu expoente máximo, o culto imperial"' ${ }^{122}$.

En resumen, es en las comunidades como castella, uici o pagi donde los habitantes indígenas continuaron encomendando su tutela a las divinidades de sus antepasados, mientras que en los nuevos municipia o en las capitales de las ciuitates fueron instaurándose progresivamente, con la necesaria participación de individuos indígenas de las clases privilegiadas, las divinidades tutelares romanas.

La polarización religiosa que se observa entre unas localidades y otras guarda una relación directa con el rango, administrativo en la mayoría de los casos, de cada una de ellas. Si ya vimos anteriormente que ningún apelativo de Bandua aludía a municipia o capitales de ciuitates, fijando nuestra mirada en las zonas concretas donde aparecen testimonios de esta divinidad, se observa que prácticamente todos proceden de lugares, a menudo uici o castella, algo alejados de los principales y más romanizados centros de población.

En cuanto a las inscripciones procedentes de la provincia española de Cáceres, conocemos cuatro altares dedicados a Bandua Roudaeco, vinculado al uicus Rouda ${ }^{123}$. Tres de ellos se hallaron cerca del castro de Villavieja, al Noroeste de Trujillo, mientras el cuarto testimonio se halló varios kilómetros al Sureste de esa localidad, en el pueblo de Madroñera (Cáceres). Todos ellos proceden, por tanto, de lugares algo alejados de la ciudad más importante de la zona, Turgalium, donde no hay testimonios de Bandua, pero sí un altar ofrecido al Genius Turgalensis ${ }^{124}$.

Cuatro aras dedicadas a Bandua Apuluseaeco se hallaron en Brozas, que podría corresponder a un antiguo uicus denominado Tongobriga ${ }^{125}$.

\footnotetext{
122 MANTAS, 1993, 235.

123 Vid. supra.

124 IGLESIAS, 1986, 129-132. En cuanto a Turgalium, uid. SAIINAS, 1990, 262-263.

125 CIL II 743. Seria, por tanto, distinto al oppidum del mismo nombre ubicado en Freixo (Marco de Canavezes).
} 
Los últimos altares cacereños dedicados a Bandua se hallaron en Montehermoso y Malpartida de Plasencia, lugares que distan más de 20 $\mathrm{Km}$. de los municipios de Capera (cerca de Oliva de Plasencia) y Caurium (Coria).

De modo parecido, en todo el territorio de la ciuitas Igaeditanorum se hallaron numerosos testimonios de Bandua, pero todos lejos de la capital, en la que tenemos el ya citado templo de Marte y varios altares dedicados a este dios ${ }^{126}$. Los testimonios más cercanos a esa población son los procedentes de Bemposta y Penamacor, poblaciones situadas a más de 15 $\mathrm{Km}$. de la capital de la ciuitas ${ }^{127}$.

Existen muchas dudas acerca de las posibles capitales de ciuitates en el resto del territorio lusitano-galaico y los distintos debates siguen abiertos, por lo que las conclusiones que obtengamos están sometidas a la confirmación de las hipótesis actuales sobre cada uno de los distintos enclaves.

Si la capital de los Tapori es Castelo Branco ${ }^{128}$, debemos resaltar que el único testimonio de Bandua de la zona procede, no de allí, sino del castro de $\mathrm{S}$. Martinho, posible uicus a unos $10 \mathrm{Km}$. de Castelo Branco ${ }^{129}$. La capital de los Lancienses Transcudani permanece ilocalizada, aunque Alarcão propuso que podría haber sido Ocelum, situada en Teixoso ${ }^{130}$. Uno de los testimonios de Bandua en este área procede del posible vicus ubicado en Orjais ${ }^{131}$; los dos restantes se hallaron en Salgueiro y Capinha enclavados, en consecuencia, a una cierta distancia de la posible capital.

El centro administrativo de los Araui se ubicó probablemente en Marialva, como parece demostrar una dedicación a Adriano por la ciuitas Arauorum ${ }^{132}$. Los altares dedicados a Bandua en esta zona cumplen el mismo criterio que en las regiones ya vistas, puesto que en este ámbito proceden de Longroiva, que sería un castellum distante 8

${ }_{126}$ MANTAS, en este sentido, llamaba la atención sobre la inexistencia de cultos indígenas en Bobadela o en la capital de los Igaeditani (1993, 235).

127 ALARCĀO, 1990, 28.

28 En un primer momento, Alarcão opinaba que podría corresponder a un uicus (1988, 11,76 $\mathrm{n}{ }^{\circ} 488$ ), pero posteriormente se inclinó a considerar que en Castelo Branco estaba la capital de la ciuitas.

129 Según ALARCĀO, aquí habría un uicus o un santuario.

130 Alarç̄o, 1990, 29.

131 ALARCÁO sugiere la existencia de un uicus en esta localidad por la existencia de algunos restos materiales romanos. Además, está muy cercana de un castro. Es posible, además, que esté visible el podium del templo dedicado a Bandua (ALARCĀO, 1988, II, 68, n. ${ }^{\circ} 331$ ).

${ }_{132}$ Alarç̄o, 1988, II, 54 n. ${ }^{\circ} 66$. 
$\mathrm{Km}$. de la capital ${ }^{133} \mathrm{y}$, probablemente, de otro uicus situado en Freixo de Numão ${ }^{134}$.

En los territorios pertenecientes a los municipios de Conimbriga, Aeminium y Bobadela no conocemos testimonios seguros de Bandua, aunque sí en la región de Viseu, posible capital de los Interannienses ${ }^{135}$. Los altares dedicados a Bandua en esta zona proceden de Queiriz (Fornos de Algodres), Insua (Esmolfe, Penalva do Castelo) y en el posible uicus situado en "Castelo do Mau Vizinho" (Sul, S. Pedro do Sul). Además, es posible la existencia de un castellum Araocelum en el área de $\mathrm{S}$. Cosmado (Mangualde), de donde procedería la pátera dedicada a Band(..) Araugel(...) ${ }^{136}$. Finalmente, los dos testimonios de Bandua hallados en Vila da Feira (Aveiro) procederían, probablemente, de un castellum indígena ${ }^{137}$ y el hallado en Cova da Lua (Espinhosela, Bragança), estaria algo distante de Castro de Avellãs, donde se ubicaría la capital de la ciuitas Zoelarum ${ }^{138}$.

En el resto del territorio portugués escasean los datos alusivos a $B a n d u a$, por lo que no se pueden obtener conclusiones relevantes a partir de los mismos. Al sur de Galicia, principalmente en la provincia de Orense, volvemos a encontrar una mayor concentración de testimonios. Uno de ellos procede de Retorta (Laza), localidad que dista más de $15 \mathrm{Km}$. de la posible capital de la ciuitas Limicorum, en el lugar llamado A Cibdá, sobre el Monte do Viso (Nocelo da Pena, Sarreaus) ${ }^{139}$. Un segundo exvoto, dedicado a Bandua Veigebreaego procede de Rairiz de Veiga; el epíteto podría aludir al nombre de la antigua localidad ${ }^{140}$ situada en el centro de un triángulo formado por tres capitales de ciuitas: la ya citada de los Limici, la de los Coelerni, posiblemente ubicada en Castromao (Celanova) y llamada Coeliobriga ${ }^{141}$ y la de los Querquerni, en la zona de Bande ${ }^{142}$. El altar ofrecido a Bandua Lansbricae, como ya vimos, se halló cerca del castro de San Ciprián de Las, cuyo nombre medieval era Laans y aludiría también a un castellum indígena ${ }^{143}$.

\footnotetext{
133 Alafcāo, 1988, II, 55, n. ${ }^{\circ} 71$.

134 Para AlARCÃo, sería un vicus de considerable importancia $\left(1988,11,53, n{ }^{\circ} 34\right)$.

135 AlARCÁO, 1990, 27.

136 Vid. supra.

137 SOUSA, 1947, 52 ss.

138 Alarcáo, 1988, II, pág. 40, 2/22.

139 TranOY, 1981, 70-71.

140 Vid. supra.

141 Tranoy, 1981, 66.

142 TRANOY, 1981, 72-73

143 Vid. supra.
} 
Finalmente, algunas de las menciones de epítetos derivados de poblaciones en altares donde no aparece el teónimo se hallaron en contextos análogos a la mayoría de los testimonios de Bandua. En este sentido, la inscripción referente al supuesto Brigo apareció en el castro situado en el monte de S. Miguel-o-Anjo (Delães, Vila Nova de Famalicão) ${ }^{144}$ y el exvoto dedicado a Tameobrigo, según Sarmento, procedería de un casteIlum indígena ubicado en el lugar llamado Castelo de Baixo ${ }^{145}$. En cuanto a Durbedico, es clara su vinculación al castellum Durbede ${ }^{146}$.

\section{CONCLUSIONES.}

La vinculación de Bandua a los Genios romanos o a Marte vendría dada por su carácter tutelar de las poblaciones indígenas, función que aún conservaría reminiscencias del papel que, probablemente, en los momentos previos a la conquista romana habría desempeñado como dios defensor de la comunidad en tiempos de guerra ${ }^{147}$.

Bajo esta óptica podríamos comprender algunos datos dispersos de la epigrafía votiva hispana. Conocemos una inscripción relativa a un Genio Defensori, procedente de Castelo do Mau Vizinho, en la feligresía de Sul (S. Pedro do Sul, Viseu) en que varios autores leian Depenori ${ }^{148}$. García planteó posteriormente dudas a esa lectura a partir de la fotografía publica$\mathrm{da}$, sugiriendo que en la segunda línea la pretendida $p$ fuera una $f y$, finalmente, que en la tercera línea hubiera una letra antes de la $0^{149}$. A la vista de dicha fotografía, nos parece que la interpretación defensori es correcta.

Ello nos lleva a plantear la siguiente teoría: dado que en un mismo recinto castreño se halló una dedicación a Bandua Ocel... y otra a un Genio defensor $y$, habida cuenta de que ambas divinidades eran frecuentemente

144 SARMENTO, $1933,301$.

145 Ibid., 1933, 308.

146 Vid. supra.

147 Cabe recordar que algunos de los santuarios prerromanos de las Galias sobre los que se puede presumir un culto relacionado con la guerra se situaban en el interior de los oppida, como Gournay-sur-Aronde o Ribemont-sur-Ancre (BRunaux, 1986, 18-21), donde se hallaron grandes depósitos de osamentas y armas. También algunos santuarios celto-ligures, como Entremont o Roquepertruse, donde no se documentan depósitos de armas pero donde si se hallaron cráneos humanos expuestos y estatuas de guerreros, se ubicaban en el interior de los oppida (ibid. 41 ss.), al igual que otros menos documentados como Zavist, Trisov o Liptovska Mara, en territorio checo (ibid., 43-44).

148 Moreira de Figueiredo leía Genio / Depenori $(1953,160)$. Esta lectura la aceptaba EnCARNAÇÃo $(1975,190)$.

149 GARCIA, 1991, 356-357 n. 204. 
vinculadas a este tipo de enclaves, podrían ser invocaciones a un mismo dios citado, por una parte, con su nombre indígena y, por la otra, mediante una de sus interpretaciones romanas. Si esta tesis es correcta, tendríamos aquí uno de los perfiles más concretos del tipo de protección que se atribuía a Bandua en el mundo indígena lusitano: defensor del recinto urbano $y$, por tanto, de la comunidad residente en él ${ }^{150}$.

La información de que disponemos nos lleva, por tanto, en la dirección de considerar a Bandua como un dios al que se adjudicaron en época pre-romana unas funciones más relacionadas con el ámbito público que con el privado, más relacionadas con la comunidad ciudadana que con grupos gentilicios; en definitiva, unas funciones de un determinado carácter político.

Estas divinidades que protegían los enclaves indígenas, aludidas como Bandua o con otro teónimo se mantuvieron, después de la conquista romana sólidamente en comunidades como castella, uici o pagi, es decir, en comunidades que habían perdido su rol político, pero en los grandes oppida, municipia o sedes de las ciuitates la influencia cultural romana, promovida por el Estado romano y asumida e impulsada por determinados elementos de las clases dirigentes indígenas, determinaba la sustitución paulatina de las divinidades comunitarias locales por determinados dioses romanos que pudieran cumplir un papel semejante como los Genios ciudadanos o, principalmente en las Galias, Marte.

Ahora bien, si son correctas estas hipótesis, cabe cuestionarse el carácter indígena de algunos de los Genios con epítetos de ciudades. En este sentido, Alarcão, Etienne y Fabre advertían que las dedicaciones al Genius Laquiniesis y al Genius Tongobrigensium las hicieron dos individuos de nombre Flavius, que se habrían beneficiado de la promoción jurídica otorgada por Vespasiano ${ }^{151}$. Si estos personajes romanizados hacian ofrendas en municipia o capitales de una ciuitas a divinidades públicas utilizando un teónimo romano nos parece razonable afirmar que estaríamos ante pruebas de un culto romano, sin perjuicio de que su consolidación se hubiera visto favorecida previamente por la existencia de una tradición religiosa indígena de análoga tipología.

Paralelamente a la anulación del poder político de los castella y la centralización del mismo en determinados oppida, el carácter público y guerrero de divinidades como Bandua fue perdiendo gran parte de su sentido

150 Cabe resaltar los sacrificios que se ofrecieron al Genius populi Romani en el 218 a.C., con ocasión de los prodigios que anunciaban la gravedad de la Segunda Guerra Púnica (Liv. XXI, 62, 9).

151 AlARCÃo et al., 1969, 232. 
conservando un carácter de dios tutelar de los individuos residentes en los uici, pagi y castella entendidos ya, únicamente, como grupo social. Sin embargo, el hecho de que en época alto-imperial se continuaran haciendo ofrendas a estos dioses con sus apelativos de poblaciones indígenas indica, a nuestro juicio, que todavía quedaba en la mentalidad colectiva de los habitantes de estas localidades una cierta conciencia del papel que sus antepasados habian atribuido a divinidades como Bandua.

\section{BIBLIOGRAFIA}

Alarcào, J.; Portugal Romano. Lisboa, 1974.

- Roman Portugal. Warminster, 1988.

- "Geografía política y religiosa da civitas de Viseu», Actas do / Col. Arq. de Viseu, Viseu, 1989, págs. 305-314.

- “Identificação das cidades da Lusitânia portuguesa e dos seus territórios", en Les villes de Lusitanie Romaine. Hiérarchies et territoires. París, 1990, págs. 21-34.

AlARCÁo, J.,R. Etienne y G. FABRE; "Le culte des Lares a Conimbriga (Portugal)», CRAl, 1969, págs. 213-236.

AlbeRTOS, M.L. y M.P. BENTO; «Testemunhos da ocupãçao romana na Região de Meimoa (Beira Baixa)", Actas XIV CNA. Vitoria, 1975, págs. 1.197-1.208.

AlBeRTOS, M.L.; “Organizaciones suprafamiliares en la Hispania antigua», BSAA 40-41, 1975, págs. 5-66.

- "Perduraciones indigenas en la Galicia romana: los castros, las divinidades y las organizaciones gentilicias en la epigrafía", Actas del Coloquio Internacional sobre el Bimilenario de Lugo, Lugo, 1977. págs. 17-26.

- "A propósito de algunas divinidades lusitanas (Arantius Ocelaecus, Arantia Ocelaeca) y el elemento ocelum", Symbolae Ludovico Mitxelena oblatae, Vitoria, 1985, págs. 469-494.

ALMEIDA, F.; “Igaedus, Divindade Lusitana, e a Senhora do Almortão", RFLL (3. ${ }^{\text {a }}$ serie) 8, 1964, págs. $65-73$.

- «Mais divindades lusitanas do grupo Band», $R F L L$ (3..a serie) 9, 1965, págs. $19-31$.

BANDEIRA, F., J.M. AlmEIDA Y J. D'ENCARNAÇÃO; "Uma árula a Banduaetobricus", Conímbriga 15, 1976, págs. 139-142.

BarRUOL, G.; “Mars Nabelcus et Mars Albiorix», Ogam 15, 1963, págs. 345-368.

BELTRÁN LLORIS, M.; “Aportaciones a la epigrafía y arqueología romana de Cáceres", Caesaraugusta 39-40, 1975-76, págs. 76-96.

BENOIT, F.; Cimiez, la ville antique (monuments, histoire). París, 1977

BINSFELD, W., K. GOETHERT-POLASCHEK y L. SCHWINDEN; Katalog der römischen Steindenkmäler des Rheinischen Landesmuseums Trier. Mainz am Rhein, 1988.

BLANCO, A.; «Pátera argéntea com representação de uma divindade lusitana», RG 69, 1959 , págs. 453-459.

BlÁZQUEZ, J.M.; Religiones primitivas de Hispania l. Fuentes literarias y epigráficas. Madrid, 1962.

BrunauX, J.L.; Les Gaulois. Sanctuaires et rites. París, 1986.

CARDozo, M.; Catálogo do Museu de Arqueología da Sociedade Martins Sarmento I. Secção lapidar e de escultura. Guimaraes, 1935.

CoIxaO, A. y J. D'EnCARnaçÃo; «Foz Coa romana. Notas epigráficas", Vila Nova de Foz Coa, 1977.

Curado, F.P.; "Ara votiva de Longroiva (Meda, Conventus Scallabitanus)» FE 11, $1985, \mathrm{n} .^{\circ} 44$.

CURCHIN, L.A.; "Vici and pagi in roman Spain", REA 87, 1985, págs. 327-343.

DE HOZ, J.; "La religión de los pueblos prerromanos de Lusitania", Manifestaciones religiosas en la Lusitania. Cáceres, 1986 , págs. 31-49.

DIAS, L.A.T.; "Necrópoles no territorium de Tongobriga", Conimbriga 32-33, 1993-1994, págs. 107-136. 
ENCARnAÇÃO, J.; "Lápides a divindades indígenas no museu de Guimarâes", RG 80, 1970, págs. 207-238.

- Divindades indigenas sob o dominio romano en Portugal. Lisboa, 1975.

- “Additamentum», Conimbriga 15,1976, págs. 142-146.

- Inscrições romanas do conventus Pacensis. Coimbra, 1984.

- "Divindades indígenas da Lusitania», Conímbriga 26, 1987, págs. 5-37.

- "Ara votiva identificada em Avis (Conventus Pacensis)", FE 46, 1994, n. ${ }^{\circ} 206$.

ETIENNE, R.; "Culte de la ciuitas - culte des pagi dans les Trois Gaules", Religio deorum. Actas del Coloquio Internacional de Epigrafía ("Culto y Sociedad en Occidente"). Sabadell, 1993, págs. 171-176.

Etienne, R., G. Fabre y P. y M. LÉvéque; Fouilles de Conimbriga II. Epigraphie et Sculpture. Paris, 1976.

FARIÑA, F.; “Dos notas a propósito de Castromao (Celanova, Orense), CEG 34, 1991, págs. 57-71.

FiguelRedo, M.; "Subsídios para o estudo da viação romana das Beiras", Beira Alta 12, 1953 , págs. 153-186.

Fleuriot, L.; “Notes sur le celtique antique», Etudes Celtiques 19, 1982, págs. 121-128.

GaRcia, J.M.; Religioes antigas de Portugal. Aditamentos e observaçoes as "Religioes da Lusitania" de J. Leite de Vasconcelos. Lisboa, 1991.

Garcia Martínez, S.M.; "Ara votiva romana de San Pedro de Trones (León)", HA 22, 1998, págs. 325-331.

HeICHELHEIM, F.; "Mars keltisch", Paulys Realencyclopädie der Classischen AltertumsWissenschaft, XIV.2. Stuttgart, 1930, 1937-1963.

- "Mercurius keltisch und germanisch", Paulys Realencyclopädie der Classischen Altertums-Wissenschaft, XV.1. Stuttgart, 1931, 982-1016.

IgLESIAS GIL, J.M.; "Genius Turgalensis", Manifestaciones religiosas en la Lusitania. Cáceres, 1986, págs. 129-132.

JuLlian, C.; Histoire de la Gaule (8 tomos). París, 1908-1926.

Laguerre, G.; “Inscriptions antiques de Nice-Cimiez (Cemelenum, ager Cemelenensis)", París, 1975.

LAMBRECHTS, P.; Contributions à l'étude des divinités celtiques. Brugge, 1942.

LEITAO, M. Y L. BARATA; «Inscrições romanas de Bemposta - Penamacor (Beira Baixa)», TAEP $23.4,1980$, págs. $627-634$.

LEITAO, M.; "Contributo para o estudo das divindades indígenas da Beira Baixa no periodo romano - duas novas aras", Trebaruna 1, 1981, págs. 51-58.

Le Roux, P. y A. Tranor; “Notes d'epigraphie romaine de Galice”, CEG 28, 1973, págs. 221-234.

LE Roux, P.; "Vicus et castellum en Lusitanie sous l'empire", SHHA 10-11, 1992-1993, págs. $151-160$.

LONGNON, A.; Les noms de lieu de la France; leur ogigine, leur signification, leurs transformations. París, 1968.

LORENZO, J. y F. BOUZA; «Inscripciones romanas votivas de la provincia de Orense», CEG 20 , 1965, pág. 127ss.

MANTAS, V. GIL; "Evergetismo e culto oficial: o constructor de templos C. Cantius Modestinus", Religio deorum. Actas del Coloquio Internacional de Epigrafia ("Culto y Sociedad en Occidente»). Sabadell, 1993, págs. 171-176.

MENOR, M.; "Otra ara al dios Tutela", Boletín Auriense 9, 1979, págs. 305-310.

Olivares, J.C.; "El dios indígena Bandua y el rito del Toro de San Marcos", Complutum 8, 1997, 205-220.

OLMSTED, G.S.; The Gods of the Celts and the indo-europeans. Budapest, 1994.

Pereira Menaut, G.; "Los castella y las comunidades de Gallaecia", Zephyrus 34-35, 1982, págs. 249-267.

- Corpus de inscripciones romanas de Galicia I (La Coruña). Santiago de Compostela, 1994.

RamiRez SÁdABA, J.L.; "Teónimo y antropónimos nuevos en la provincia de Lusitania y zonas próximas", Act. $V$ Col. Leng. y Cult. Prerromanas de la Península lbérica, Salamanca, 1989, págs. 425-449.

ReINACH, S.; Cultes, Mythes et Religions (tomo III). Paris, 1913.

RIVAS, J.C.; «Nuevas aras romanas orensanas y rectificaciones interpretarivas en torno a otros epígrafes galaico-romanos ya conocidos", Boletín Auriense 3, 1973, págs. 57-96. 
RIVET, A.L.F. y C. SMITH, The place-names of Roman Britain. London, 1979.

Rodriguez Colmenero, A.; Aquae Flaviae /. Fontes epigráficas. Chaves, 1987.

RodRiguez GonzALEZ, X.; "Nueva aportación al panteón galaico-romano: Lari Ocaelaego", Boletín Auriense 20-21, 1990-1991, págs. 204-209.

SALINAS, M.; "Las ciudades romanas de Lusitania oriental: su papel en la transformación del territorio y la sociedad indígena", en Les villes de Lusitanie Romaine. Hiérarchies et territoires. París, 1990, págs. 255-263.

Santos, L., P. Le Roux y A. Tranoy, «Inscrições romanas do Museu Pio XII em Braga", BA 37 , 1983, págs. 183-305.

SARMENTO, F.M.; «Inscrições inéditas», Dispersos. Coimbra, 1933, págs. 175-180. - "Para o panteao lusitano"; Dispersos. Coimbra, 1933, págs. 297-308.

SAYAS ABENGOCHEA, J.J.; "El territorio aquitano entre la asimilación romana y el mantenimiento de su especificidad", Segundo Congreso General de Historia de Navarra. Príncipe de Viana, anejo 14, 1992, págs. 153-180.

SousA, A.; "Vila da Feira Lusitano-Romana", Douro Litoral 8, 1947, págs. 52-59.

Speidel, M.P., y A. Dimitrova-MILCEVA, "The cult of the Genii in the Roman Army and a New Military Deity», ANRW Il-16.2, 1978, págs. 1543-1551.

TABOADA, J.; "Ara romana de Villaza (Verin)", Bol. Mus. Arq. Orense 5, 1949, págs. 55 ss.

THEVENOT, E.; Sur les traces des Mars celtiques (entre Loire et Mont-Blanc). Brugge, 1955.

Tranoy, A.; La Galice romaine. Recherches sur le nord-ouest de la péninsule iberique dans l'antiquité. París, 1981.

- «Enigmes epigráphiques et nouveaux cultes indigènes dans le conventus de Braga», RG 94, 1984, págs. 443-449.

- "L'organisation urbaine dans le conventus Scallabitanus", en Les villes de Lusitanie Romaine. Hiérarchies et territoires. París, 1990, págs. 11-20.

Vasconcelos, J. LeIte de; Religioes da Lusitania II (1905) y III (1913). Lisboa.

VAZ, J.L.; "Fragmento de ara do Museu de Grao Vasco (Viseu)", FE 31, 1989, n. 140.

- A civitas de Viseu. Espaço e sociedade. Viseu, 1993. 